\title{
$\mathrm{Yb}_{9+\mathrm{x}} \mathrm{CuMg}_{4-\mathrm{x}}(\mathrm{x}=\mathbf{0 . 0 3 4})$ : a kappa-phase formed by Lanthanoids
}

\author{
Serena De Negri ${ }^{1}$, Vitaliy Romaka ${ }^{2}$, Pavlo Solokha ${ }^{1}$, Adriana Saccone ${ }^{1}$, Gerald Giester ${ }^{3}$, \\ Herwig Michor ${ }^{4}$ and Peter F. Rogl ${ }^{5, *}$ \\ ${ }^{1}$ Dipartimento di Chimica e Chimica Industriale, Università di Genova \\ Via Dodecaneso 31, I-16146 Genova, Italy \\ ${ }^{2}$ Department of Materials Science and Engineering, Lviv Polytechnic National University, \\ Ustiyanovycha Str. 5, 79013 Lviv, Ukraine \\ ${ }^{3}$ Institute of Mineralogy and Crystallography, University of Vienna, \\ Althanstrasse14, A-1090 Wien, Austria \\ ${ }^{4}$ Institute of Solid State Physics, TU Wien,
}

Wiedner Hauptstraße 8-10, A-1090 Wien, Austria

${ }^{5}$ Institute of Materials Chemistry and Research, University of Vienna,

Währingerstraße 42, A-1090 Wien, Austria 


\section{Crystal structure of $\mathrm{Yb}\left(\mathrm{Cu}_{0.99} \mathrm{Mg}_{0.01}\right)$ with $\mathrm{FeB}$-type}

The alloy $\mathrm{Yb}_{30} \mathrm{Cu}_{40} \mathrm{Mg}_{10}$ (at.\%, as cast) yielded a single crystal, the diffraction pattern of which was completely indexed on an orthorhombic unit cell $(a=7.5689(3) \mathrm{nm}, b=4.2597(1) \mathrm{nm}, \mathrm{c}=5.7703(2)$ $\mathrm{nm})$, which together with the observed extinctions $((0 \mathrm{k} \ell)$ extinct for $\mathrm{k}+\ell=2 \mathrm{n}+1$ and $(\mathrm{hk} 0)$ for $\mathrm{h}=$ $2 \mathrm{n}+1$ ) indicates the $\mathrm{YbCu}$-phase with the FeB-type structure (space group Pnma). Least squares refinement confirmed the structure type. EMPA data obtained from the as cast alloy define a small solubility of $\mathrm{Mg}(\sim 1 \mathrm{at} . \%)$ in $\mathrm{YbCu}$. Consistent with lattice parameters, which appeared slightly enhanced with respect to the known binary $\mathrm{YbCu}$, we can assume a small random substitution of 1 at. $\% \mathrm{Mg}$ in the $\mathrm{Cu}$-sites. A final refinement slightly lowered the $\mathrm{R}$-value reaching $\mathrm{R}_{\mathrm{F}}=0.0195$ (for $420 \mathrm{~F}_{\mathrm{o}}>4 \sigma\left(\mathrm{F}_{\mathrm{o}}\right)$ of a total of 492 reflections) with marginal residual electron densities of $\left.2.7 \mathrm{e}^{-} / \AA^{3}\right)$. For details see Table S1.

Table S1. X-Ray single crystal data for $\mathrm{Yb}\left(\mathrm{Cu}_{0.99} \mathrm{Mg}_{0.01}\right)$ (room temperature, $\omega$-scans, scan width $2^{\circ}$; redundancy $>7$ ); structure standardized with program Structure Tidy.

\begin{tabular}{|c|c|}
\hline Parameter / compound & $\mathbf{Y b C u}$ \\
\hline Formula from EPMA (at.\%) & $\mathrm{Yb}\left(\mathrm{Cu}_{0.99} \mathrm{Mg}_{0.01}\right)$ \\
\hline Formula from refinement & $\mathrm{Yb}\left(\mathrm{Cu}_{0.99} \mathrm{Mg}_{0.01}\right)$ \\
\hline Single crystal size & $40 \times 60 \times 54 \mu \mathrm{m}^{3}$ \\
\hline Structure type / space group & FeB / Pnma \\
\hline $\begin{array}{l}\text { Lattice parameters, a,b,c } \\
{[\mathrm{nm}]}\end{array}$ & 7.5689(3), 4.2597(1), 5.7703(2) \\
\hline$\mu_{\text {abs }}\left[\mathrm{mm}^{-1}\right]$ & 58.11 \\
\hline $\begin{array}{l}\text { Data collection, } 2 \Theta \\
\operatorname{range}\left({ }^{\circ}\right)\end{array}$ & $2 \leq 2 \Theta \leq 72.5 ; 110 \mathrm{sec} /$ frame \\
\hline Total number of frames & $210 ; 5$ sets \\
\hline Reflections in refinement & $420 \geq 4 \sigma\left(F_{o}\right)$ of 492 \\
\hline Mosaicity & $<0.45$ \\
\hline Number of variables & 14 \\
\hline $\mathrm{R}_{\mathrm{F}}=\Sigma \mid \mathrm{F}_{0}-\mathrm{F}_{\mathrm{c}} / \Sigma \mathrm{F}_{0}$ & 0.0195 \\
\hline $\mathrm{R}_{\text {Int }}$ & 0.055 \\
\hline wR2 & 0.045 \\
\hline GOF & 1.15 \\
\hline Extinction (Zachariasen) & $0.0177(9)$ \\
\hline Atom site 1 & $\begin{array}{l}\text { 1.0(-) Yb in } 4 c(x, 1 / 4, z) \\
x=0.17892(3) ; z=0.12715(4)\end{array}$ \\
\hline $\mathrm{U}_{11} ; \mathrm{U}_{22} ; \mathrm{U}_{33}\left[\right.$ in $\left.10^{2} \mathrm{~nm}^{2}\right]$ & $0.0114(1) ; 0.0113(1) ; 0.0115(1)$ \\
\hline $\begin{array}{l}\mathrm{U}_{12}=\mathrm{U}_{23}=0 ; \mathrm{U}_{13} ;[\mathrm{in} \\
\left.10^{2} \mathrm{~nm}^{2}\right]\end{array}$ & $0.0012(7)$ \\
\hline Atom site 2 & $\begin{array}{l}\mathbf{0 . 9 9 C u}+\mathbf{0 . 0 1} \mathrm{Mg} \text { in } 4 \mathrm{c}(\mathrm{x}, 1 / 4, \mathrm{z}) \\
\mathrm{x}=0.0319(1) ; \mathrm{z}=0.6128(1)\end{array}$ \\
\hline $\mathrm{U}_{11} ; \mathrm{U}_{22} ; \mathrm{U}_{33}\left[\right.$ in $\left.10^{2} \mathrm{~nm}^{2}\right]$ & $0.0134(3) ; 0.0111(3) ; 0.0138(3)$ \\
\hline $\begin{array}{l}\mathrm{U}_{12}=\mathrm{U}_{23}=0 ; \mathrm{U}_{13} ;[\mathrm{in} \\
\left.10^{2} \mathrm{~nm}^{2}\right]\end{array}$ & $0.0002(2)$ \\
\hline
\end{tabular}


Table S2. Anisotropic thermal displacement parameters $U i j\left(\mathrm{~nm}^{2} \times 10^{2}\right)$ for $\mathrm{Yb}_{9+\mathrm{x}} \mathrm{CuMg}_{4-\mathrm{x}}$

\begin{tabular}{|l|l|l|l|l|l|l|}
\hline Atom & $U_{11}$ & $U_{22}$ & $U_{33}$ & $U_{23}$ & $U_{13}$ & $U_{12}$ \\
\hline $\mathrm{Yb} 1(12 k)$ & $0.0214(1)$ & $0.0225(1)$ & $0.0216(1)$ & $-0.00271(9)$ & $-0.00135(5)$ & $0.0112(1)$ \\
\hline $\mathrm{Yb} 2(6 h)$ & $0.0202(1)$ & $0.0281(2)$ & $0.0255(2)$ & 0.000 & 0.000 & $0.0141(1)$ \\
\hline $\mathrm{Yb} 3+\mathrm{Mg} 1(2 a)$ & $0.021(1)$ & $0.021(1)$ & $0.021(2)$ & 0.000 & 0.000 & $0.0105(7)$ \\
\hline $\mathrm{Mg} 2(6 h)$ & $0.020(1) ;$ & $0.024(1)$ & $0.014(1)$ & 0.000 & 0.000 & $0.0118(7)$ \\
\hline $\mathrm{Cu}(2 c)$ & $0.0340(7)$ & $0.0340(7)$ & $0.0216(9)$ & 0.000 & 0.000 & $0.0170(3)$ \\
\hline
\end{tabular}

Table S3. Crystallographic data of $\mathrm{Yb}_{9} \mathrm{CuMg}_{4}\left(P 6_{3} / m m c\right)$ after geometry optimization (DFT)

\begin{tabular}{|l|l|l|l|}
\hline Compound & \multicolumn{2}{|l|}{$\mathrm{Yb}_{9} \mathrm{CuMg}_{4}$} \\
\hline $\begin{array}{l}\text { Lattice parameters } \\
(\mathrm{nm})\end{array}$ & \multicolumn{2}{|l|}{$0.9963580, c=1.0013874 \mathrm{~nm}$} \\
\hline Atoms & $x / a$ & $y / b$ & $z / c$ \\
\hline $\mathrm{Yb} 1$ & 0.204329 & 0.408658 & 0.057237 \\
\hline $\mathrm{Yb} 2$ & 0.539484 & 0.078969 & $1 / 4$ \\
\hline $\mathrm{M}(\mathrm{Mg} / \mathrm{Yb})$ & 0 & 0 & 0 \\
\hline $\mathrm{Cu}$ & $1 / 3$ & $2 / 3$ & $1 / 4$ \\
\hline $\mathrm{Mg} 2$ & 0.893360 & 0.786720 & $1 / 4$ \\
\hline
\end{tabular}



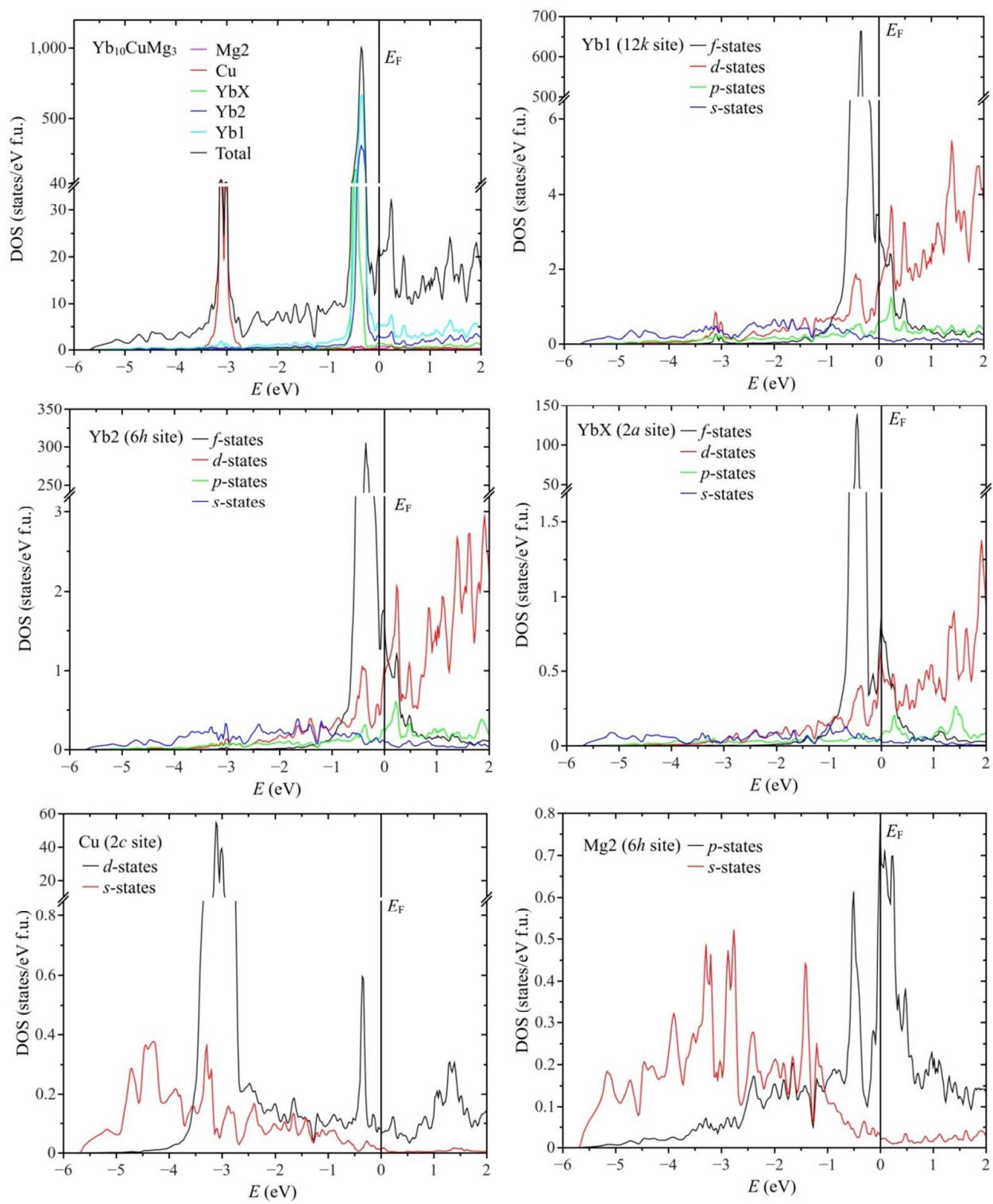

Figure S1. Distribution of the total and partial density of states (per formula unit) of $\mathrm{Yb}_{10} \mathrm{CuMg}_{3}$. The Fermi level $E_{\mathrm{F}}$ is located at $E=0 \mathrm{eV}$. 